\title{
On the Development of a Universal Two-channel Sensitive Digital Sensor for Recording Seismic Vibrations
}

\author{
V. S Potylitsyn \\ Institute of Radio Technology \\ Siberian Federal University \\ Krasnoyarsk, Russia \\ markuss86@mail.ru
}

\author{
O. A Maikov \\ Military Engineering Institute \\ Siberian Federal University \\ Krasnoyarsk, Russia \\ maykov_oleg@bk.ru
}

\author{
D. S. Kudinov \\ Institute of Radio Technology \\ Siberian Federal University \\ Krasnoyarsk, Russia \\ kudinovdanil@yandex.ru
}

\begin{abstract}
This article discusses the development of twochannel sensitive sensors for recording seismic vibrations. The influence of the instability of the element base in the implementation of sensor data using analog amplifiers and filters is considered. It is shown that the effect of this instability can lead to the formation of measurement errors. Even a change in the cutoff frequency of several hertz leads to the formation of an error of the order of $10-15 \%$. The characteristics of digital filters that can be used in these sensors are given, which, using modern microcontrollers of the ARM architecture, will allow not only filtering in real time, but also perform mathematical processing of received signals.
\end{abstract}

Keywords - sensor, ARM, digital filtering, dual channel, seismic, acoustic.

\section{INTRODUCTION}

Currently, there is an acute problem of implementing digital systems using analog sensors for registering analog signals of various natures; this problem is solved either using high-resolution ADCs or using various combinations of analog amplifiers and then converting them to digital form and subsequent filtering. The received signals can be analog signals converted from various sensors, such as radar, analog thermometers, seismic sensors, and so on.

Each method has its own advantages and disadvantages. Using an ADC with a large number of digits (about 24) is associated with a very complex power supply stabilization scheme. Implementation of circuits with an ADC with such bit width for low (up to $400 \mathrm{~Hz}$ ) frequencies is not difficult due to the use of a sigma-delta ADC [1], and the use of analog amplifier stages introduces non-linear distortions in the signal, and the creation of two identical amplifier stages for a two-channel system bases of radio components are sometimes simply impossible.

Thus, the development and creation of a universal sensor with minimal frequency distortion and digital filtering is a rather complicated and urgent task. Sensors of this type are used to organize various kinds of multichannel and differential systems. The use of this type of sensors is currently possible, both for the organization of lowfrequency communications for the needs of an emergency communication channel in mine workings, and for recording seism acoustic and electromagnetic fields [2-6].

\section{ANALISYS}

The first modifications of this sensor were considered in article [7]. Here, in brief, we give a description of this circuit, the analog paths were implemented on an INA128 chip manufactured by Texas Instruments, which provides an input resistance of about $3 \mathrm{M} \Omega$, a band-rejection filter is used at a frequency of $50 \mathrm{~Hz}$ according to the $\mathrm{T}$-shaped $\mathrm{RC}$ bridge. The signal is then converted to digital form using an MSP430 microcontroller and further digital filtering of the signals takes place.

During the course of carrying out field tests of this scheme, the following disadvantages were found.

The implementation of digital filtering of signals using this microcontroller is limited by the number of links of 200 units for a filter with a finite impulse response, which often limits the creation of band-pass filters with sufficient frequency slope.

The development and application of this class of microcontrollers showed its insufficient computing power even at a clocking frequency of $24 \mathrm{MHz}$ [8]. Thus, the use of more powerful types of microcontrollers, which allow digital filtering in real time, including with floating-point numbers, is required. The authors of this article proposed to use the STM32 microcontroller, which allows to eliminate all the above disadvantages.

ARM architecture is more preferable for the implementation of this class of devices as it has all the same advantages as the MSP430 family, but with higher performance for calculations and the ability to display information on the display. It should be said that in this family ADCs of sequential approximation are used [9].

To convert the signal into digital form, it is preferable to use two ADCs with simultaneous conversion. The clocking frequency of the ADC can vary widely from a few kilohertz to tens of megahertz. The consecutive approximation ADC in the STM32 family uses 12.5 clocks to read the conversion result. In order to minimize high-frequency interference, the signal accumulation should be made maximum in duration. The maximum signal accumulation time in this case is 239.5 
cycles. Thus, the total time between samples will be equal to 252 clocks, therefore, the required clocking frequency is $64512 \mathrm{~Hz}$, the closest frequency that can be set in this MCs family for the $\mathrm{ADC}$ is $65 \mathrm{kHz}$, which is acceptable for this case.

Microcontrollers of this architecture have a wide range of models and classes, which allows modernizing equipment using more powerful and modern microcontrollers without changing the source code of the microprogram.

In real conditions, when prototyping boards and conducting various experiments, it was found that the constant measurement error introduced by the non-identity of the input analog paths during band-pass analog filtering was about $20 \%$ for the correlation function, which is not acceptable for use in real conditions.

To solve this problem, the use of specialized filter microcircuits on switched capacitors is proposed. The use of this type of filter has two applications with external clocking and using an internal oscillator, the frequency of which is set by an external capacitor. As was shown, due to the inaccuracy of the ratings of the components of the element base, the use of external capacitance is not possible. Thus, it is preferable to use external clocking, which can provide the digital part of the receiving equipment, and the use of $\mathrm{MCs}$ will allow you to flexibly adjust the cut-off frequency of these filters.

The family of microcontrollers STM32, has the ability not only to carry out calculations and display on the display, the results of calculations, but also to control the gain of the analog part of the receiving equipment via the SPI bus. Given that this family allows clocking up to $72 \mathrm{MHz}$ and has an arithmetic processor that allows floating-point calculations, the implementation of two digital filters will not be difficult.

The presence of a wide range of different external buses (UART, SPI, I2C, CAN, and others) allows you to easily select a display for this receiving equipment. Almost all modern displays are equipped with a controller for displaying data on the screen; therefore, all that is required is to ensure the interaction of these components using the required protocol and data bus.

As mentioned above, differential instrumentation amplifiers are used to realize the gain in the analog part of the receiving equipment; in this class of amplifiers, the gain is usually controlled by a single resistive element, that is, by changing the resistance of the resistor, the gain changes. In order to ensure the use of equipment in different conditions it is proposed to use digital potentiometers for gain control.

Modern electronic industry produces various modifications of these potentiometers, for this case, of interest are those that can operate at two polar power and can be controlled via UART or SPI buses. The most suitable for the implementation of this function is the digital potentiometer AD5142 / AD5142, which is essentially twochannel, which will allow you to adjust not only the gain of the preamplifiers, but also the subsequent amplification stage. The functional diagram of this chip is shown in Figure 1.

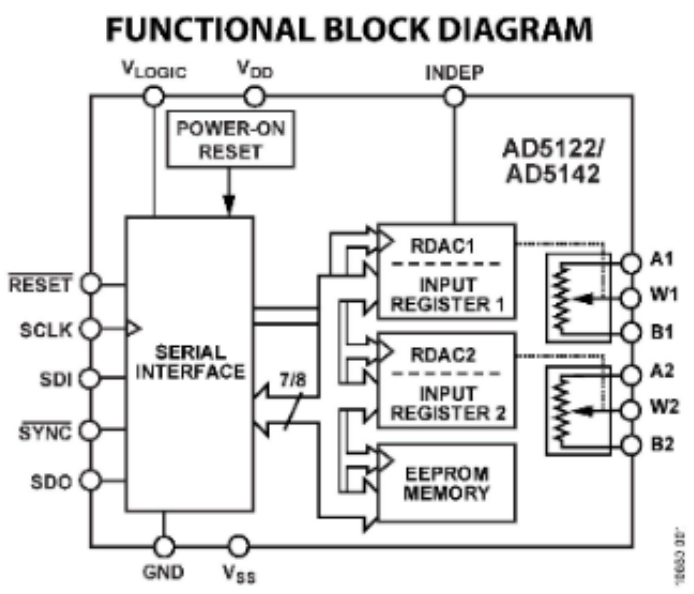

Fig. 1. Architecture AD5142 / AD5142

The AD5142 / AD5142 chips allow you to adjust the resistance in $78 \mathrm{Ohm}$ steps from 0 to $10 \mathrm{k} \Omega$, and in 380 Ohm steps from 0 to $100 \mathrm{k} \Omega$, which, using the INA128 chip, will allow you to adjust the gain from 0 to 500 per cascade, which optimal.

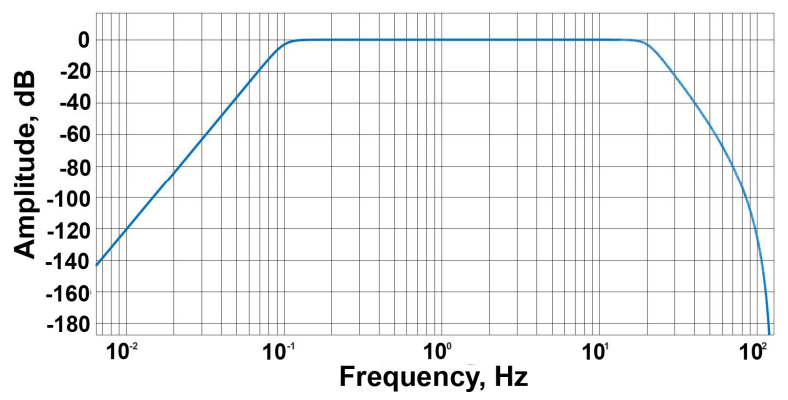

Fig. 2. Frequency response bandpass filter Butterworth 12 order

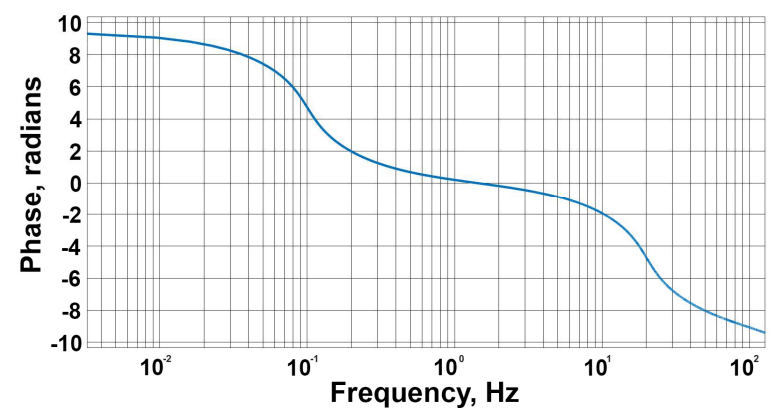

Fig. 3. Phase-frequency characteristic of the bandpass filter of the Butterworth 12 order

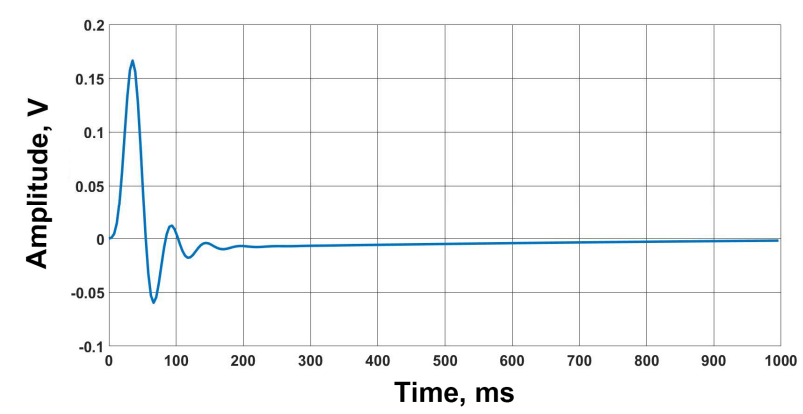

Fig. 4. The impulse response of the band-pass Butterworth 12-order filter 
As can be seen from the presented characteristics of the digital filter, the industrial frequency suppression of $50 \mathrm{~Hz}$ is provided at $50 \mathrm{~dB}$, taking into account the use of a band-stop filter, the total suppression for this frequency will be about $120 \mathrm{~dB}$, which will allow work to be done near industrial facilities and power lines.

Since the same digital filter will be used for both channels, the distortions introduced by it in the passband and cut-off frequency will be identical and will not distort the analog frequency characteristics. The settling time of this filter when exposed to impulse noise will be about $1 \mathrm{~s}$, which is acceptable for such frequency response and phase response. For operation in conditions of impulse noise, it is possible to disable this type of filtering.

To pair the receiving equipment with a personal computer, you can use the standard UART bus using a specialized UART-USB interface converter chip. At the moment there is a wide range of manufacturers chips (CP2102, FT232RL and others).

Simulation of the analog part using various analog filters is shown in Figure 5, where a second-order active RC filter with multiloop feedback was used as an output low-pass filter. This scheme allows you to allocate the desired frequency band, with the suppression of industrial interference $50 \mathrm{~Hz}$.

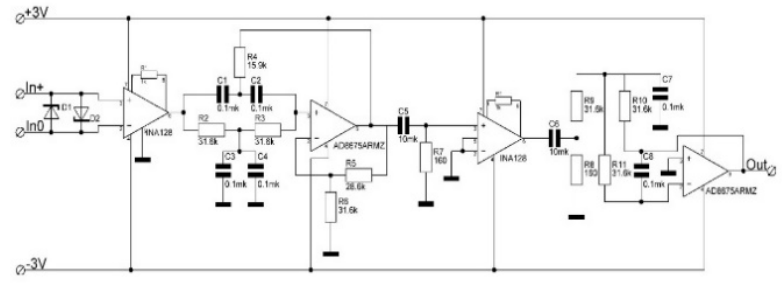

Fig. 5. Receiver circuitry.

The simulation of this circuit was carried out in the Microcap software environment, the frequency response of the circuit is shown in Figure 6, the experimental response obtained is shown in Figure 7, which shows that the calculated data almost completely agree with the data obtained during the prototyping of this circuit.

As can be seen from the figures, there is a slight deviation in the suppression of industrial interference of 50 $\mathrm{Hz}$, which is due to the inaccuracy of the radio components.

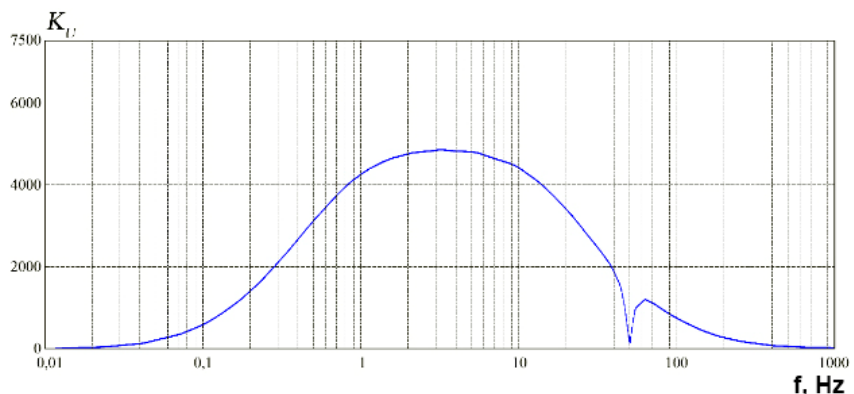

Fig. 6. The frequency response of the circuit obtained by modeling in the environment Microcap.

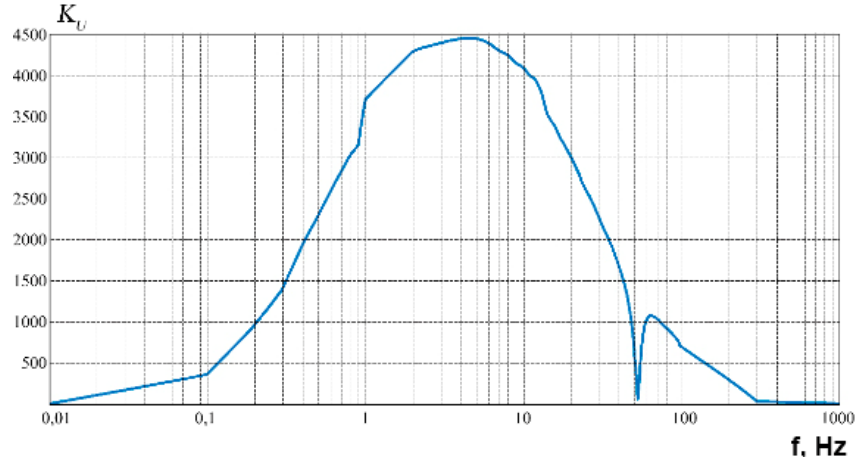

Fig. 7. Experimental frequency response obtained during the simulation

To test the effect of the non-identity of the frequency response of the channel characteristics, the following modeling was carried out in the Matlab software environment; a temporary implementation of additive white Gaussian noise was created, with 46080 samples, which at a sampling frequency of $256 \mathrm{~Hz}$ corresponded to a measurement time of 180 seconds. Further, the instability of the cut-off frequency of the low-pass filter was estimated, for the example of the calculation, a Butterworth filter of 2, 4,6 , 8 and 10 orders was taken. Thus, various displacements were modeled as the upper border of the band-pass filter as below (Figures 8-10). The figure shows the total offset of both the upper cutoff frequency and the lower one.

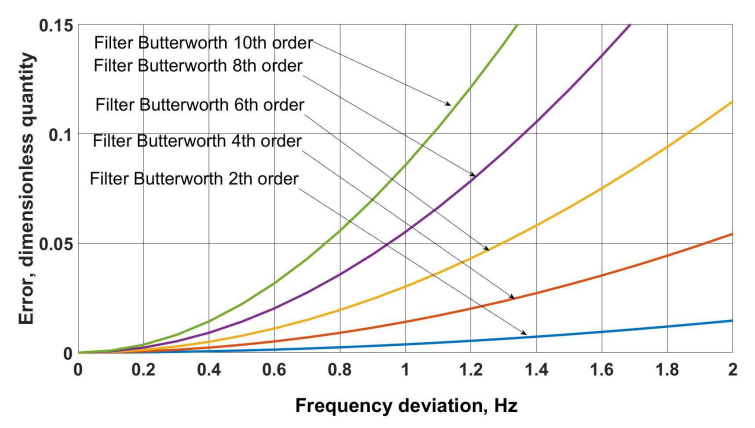

Fig. 8. Impact of cell instability using analog low-pass filters

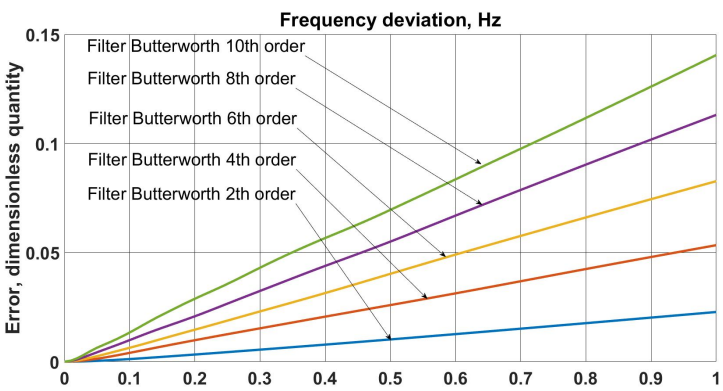

Fig. 9. Impact of cell instability using analog high-pass filters 


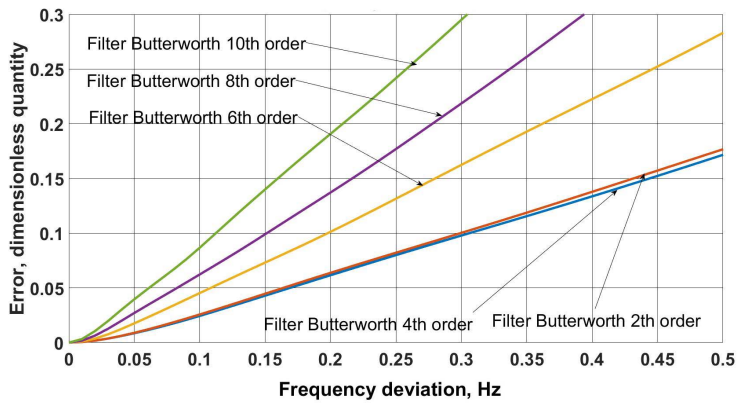

Fig. 10. Impact of cell instability using analog high-pass and low-pass filters

As can be seen from the figures, the contribution when the cutoff frequencies of the filter are different in different channels, only for the top or only for the bottom within one hertz is not so significant and does not exceed 0.15 , in this case, the normalized difference dispersion of the signal is calculated, calculated using the following formula.

$$
\eta=\frac{\frac{1}{T} \cdot \int_{0}^{T}\left(E_{1}(t)-\bar{k} \cdot E_{2}(t)\right)^{2} \cdot d t}{\sigma_{E 1}^{2}}
$$

Where, $\mathrm{T}$ is the measurement time, E1 ( t) and E2 (t) are signals in different channels, is the signal dispersion E1 $(\mathrm{t})$.

This algorithm allows the detection of inertial objects in a passive way, which is used for inverse problems.

If both the upper cut-off frequency and the lower for a second-order band-pass filter of $0.5 \mathrm{~Hz}$ deviate, this value is already equal to 0.15 . In reality, this suggests that the use of analog filtering for this algorithm is limited by the instability of the nominal electronic components.

\section{CONCLUSION}

Thus, the use of data in this article shows that it is possible to implement a two or three component sensor for seismic vibrations, which makes it possible to register oscillations, including reducing interchannel distortions. It is shown that when using analog filtering, the instability of the element base leads to the formation of measurement errors in precision calculations of up to 15 percent, which in some cases is unacceptable. Especially when registering seismoelectric or electromagnetic oscillations of low frequency.

Digital filters in this case are more relevant for such tasks, since they do not introduce distortions into the resulting error between the channels. But the use of such filters is associated with the use of sufficiently powerful microcontrollers, which in this case increases the cost of the entire device. It should be said that for this kind the most suitable would be the use of a high-bit sigma-delta ADC with a large input dynamic range, which will allow transferring signals of a digital type using any amplifying or filtering cascades with minimal distortion.

\section{ACKNOWLEDGMENT}

The authors thank the Siberian Federal University for providing the infrastructure with the implementation of this project. This work has been done with the support of Council on grants of the President of the Russian Federation (scholarships of the President of the Russian Federation to young scientists and graduate students № 904.2018.1) and supported by grant of the Russian Science Foundation (project No. 18-79-00137).

\section{REFERENCES}

[1] Maksimov L. A., Vedernikov G. V. and Yashkov G. N., Geodinamicheskii shum zalezhei uglevodorodov i passivno-aktivnaya seismorazvedka MOGT [Geodynamic noise of hydrocarbon deposit and passive-active seismic survey CDP method]. Neftegazekspozitsiya, Vol. 6, Issue 45, 2015, pp. 55-57.

[2] Berg A. E., Method of Seismo-Electromagnetic Detecting of Hydrocarbon Deposit, US Patent No. 7330790, October, 2005.

[3] Berg-Vishnyakov, A. E. [2012] Technology and Experience of Application of Electromagnetic Transient Buildup Affected by Seismic Radiation (TBSR) in the Search for Raw Hydrocarbon Deposits in conditions of shelf, Materials of the X-th international geophysical scientific-practical seminar «The application of modern electrical exploration technologies in prospecting of mineral deposits», p. 35-39., St Petersburg

[4] Dobrynin, S. I., Golovin, S. V., Bobrovnikov, L. Z., Melaev, D. V. [2012] Innovative Technology of Hydrocarbon Deposits Assessment and Monitoring in Oil and Gas Develop Reserves, Petroleum Engineer. Moscow: "IDS Dilling”' LLC, No. 2, pp. 24-29, 2012.

[5] Melnikov, V. P., Lisov, V. I., Bryukhovetski, O. S.,Bobrovnikov, L. Z. [2010] Innovative Geophysics: Binary Technologies of Direct Exploration. Part 1, Petroleum Engineer.M.: "IDS Drilling”, No. 1. pp. 47-51

[6] Trigubovich G. M. and Epov M. I., New opportunities for electromagnetic reconnaissance, Abstracts of the report of the scientific-practical conference on November 24- 26, 2008, SNIIGGiMS, Novosibirsk, pp.16-19.

[7] V. S. Potylitsyn, G. Y. Shaydurov, D. S Kudinov., Some results of field trials of induced polarization method based on the extraction of information from the natural electromagnetic field of the Earth, Devices and systems of Exploration Geophysics, Vol. EAGO, Saratov, 2012, № 4, p. 51-53

[8] Reference manual. MSP430FR4xx and MSP430FR2xx. User's Guide. 2018.

[9] Reference manual. STM32F102xx ARM-based MCUs. STMicroelectronics. 2015. 